\title{
Protective Effect of Chicken Egg Shell Powder Solution (CESP) on Artificially Induced Dental Erosion: An in Vitro Atomic Force Microscope Study
}

\author{
Sandleen Feroz ${ }^{*}$, Faisal Moeen, Saleha Nisar Haq \\ Department of Dental Materials, Islamic International Dental College, Riphah University, Islamabad, Pakistan \\ *Corresponding author: sandaleenferoz@yahoo.com
}

\begin{abstract}
Aim: The aim of this in vitro study was to evaluate the effect of chicken egg shell powder solution (CESP) on the dental erosion produced by Coca Cola drink in permanent teeth. Material and methods: Thirty extracted human incisors were collected for this study purpose. After extraction all the specimens were cleaned and sectioned longitudinally resulting in 60 samples from a total of 30 tooth samples. All samples were randomly assigned to the following three groups. Group 1: Untreated group, Group 2: Demineralization by soft cola drink (4 cycles of 2 minutes' immersion at 6 hours' interval), Group 3: Demineralization by soft cola drink (4 cycles of 2 minutes' immersion at 6 hours' interval) followed by 7 days' immersion in CESP. For surface roughness assessment images were taken from AFM. The root means square roughness (Rrms) was calculated from the AFM images. The differences in the mean roughness values between the three groups were analyzed by Anova and Tukey's post hoc test. Results: One way Anova analysis revealed a statistically significant differences with p-value of 0.00 , between the mean roughness values of three groups. Tuckey's post hoc test revealed that there is a statistically significant difference $(0.158 \pm 0.012)$ between the surface roughness of Group 2 and Group 3 as well as between Group 1 and Group 3(0.085 \pm 0.012$)$ with a p-value of 0.00 , indicating that following treatment by CESP the mean roughness values decreased significantly. Conclusion: CESP reduces the surface roughness of all treated teeth samples giving encorughment to the protective effect of CESP againts erosive enamel loss.
\end{abstract}

Keywords: chicken egg shell powder solution (CESP), Enamel Demineralization, Atomic Force Microscopy (AFM)

Cite This Article: Sandleen Feroz, Faisal Moeen, and Saleha Nisar Haq, "Protective Effect of Chicken Egg Shell Powder Solution (CESP) on Artificially Induced Dental Erosion: An in Vitro Atomic Force Microscope Study." International Journal of Dental Sciences and Research, vol. 5, no. 3 (2017): 49-55. doi: 10.12691/ijdsr-5-3-2.

\section{Introduction}

The paradigm of modern dental practice is now gradually moving towards the concept of minimal invasion dentistry(MID) which is a conservative philosophy that mainly emphasizes upon early detection of carious lesions, remineralization of tooth surfaces and preservation of surrounding tooth structure [1,2]. Along with dental caries whose prevalence is very high in many developing countries dental erosion is now considered as a major cause of dental tissues loss affecting not only adults but also children and adolescents in developed countries $[3,4,5]$.

Dental erosion is a localized chemical dissolution of tooth surfaces without bacterial involvement [6]. Although, teeth are exposed to continuous cycles of demineralization followed by remineralization, this delicate balance can easily be disturbed by extensive use of low $\mathrm{pH}$ drinks like carbonated drinks, fruit juices, acidic beverages, wines, candies etc. which leads to acidic dissolution of the inorganic phase of tooth and subsequent loss of tooth substance [7]. Since enamel lacks an inherent ability to repair itself if affected by abrasion, fracture or caries, the rebuilding of early, superficial enamel layer loss therefore depends heavily on the composition of saliva as well as on tooth pastes and creams containing alloplastic materials such as calcium or fluoride [8].

Demineralization resulting from loss of calcium and phosphate ions can be restored by using non-invasive calcium phosphate delivery system. Modern tooth pastes/creams/gels containing sodium fluoride or tri calcium phosphate have now been considered as the most effective source of in organic ions required for enamel remineralization [9].

Phospho - peptide amorphous calcium phosphate CPPACP (Recaldent TM) derived from milk products have shown promising results by forming a protective layer and enhancing the remineralization both in vitro and in vivo [10]. CPP-ACP exhibit a remarkable ability to reduce enamel erosion and has been successfully incorporated into mouth washes,sports drink and sugar free chewing gums $[6,20]$. Similarly, the use of CESP in various fields has been studied [11]. Chicken egg is a known source of Ca containing about $39 \%(\mathrm{w} / \mathrm{w})$ of elemental Ca. It not -only bolsters the chondrocyte differentiation in vitro but also increases the bone mineral density in animal model of female rat [12]. CESP also increases the bone 
mineral density and reduces bone pain in postmenopausal women and women with senile osteoporosis [11,13].

Many studies have been done to understand the effects of different remineralizing agents on early enamel carious lesions but there is still dearth of information regarding the use of CESP and its possible effects on eroded enamel surface. Demineralized tooth surfaces have been studied widely by using technique sensitive instruments such as scanning electron microscope [14]. In contrast AFM studies are faster, require minimal sample preparation and the potential drift free stage provides an extremely stable imaging platform [15].

The aim of this in vitro study is to evaluate the protective effects of CESP on enamel lesions by the aid of AFM Nano indentation.

\section{Methodology}

\subsection{Preparation of Chicken Egg Shell Powder Solution (CESP)}

The Chicken Egg Shell Powder was prepared by the calcination protocol given by World Property Intellectual organization (WO/2004/105912: Method of Producing egg shell powder) [16]. Chicken Egg Shell contains about 95\% of calcium carbonate which on conversion to basic calcium oxide due to calcination is responsible for an increase in alkalinity [17].

Twelve chicken eggs used were cleaned with distilled water and kept in hot boiling water for 10 mins at $100^{\circ} \mathrm{C}$ to facilitate the removal of membranes. The egg shells were crushed and powdered to small particles with sterile mortar and pestle. The tiny, crushed particles obtained were then kept in a muffle furnace (Neycraft Model JFF 2000) at $1200^{\circ} \mathrm{C}$ to make sure the resulting powder is pathogen free.

One gram of CESP was dissolved in $20 \mathrm{ml}$ of $4 \%$ acetic acid and the clear fluid obtained at the top of test tube was transferred to a beaker. The $\mathrm{pH}$ of the solution obtained was 11.7 which was measured by using a $\mathrm{pH}$ meter (Mettler Toledo).

\subsection{Sample Preparation}

Thirty extracted human permanent incisors were collected for this study. Following extraction they were washed to remove soft tissue debris and then put in an ultrasonic cleaner for 6 minutes followed by disinfection in a sodium hypo-chloride solution (5\%) for 1 hour. Teeth were then stored in deionized water prior to testing. The collected specimens were inspected carefully for any white spot lesions, cracks or hypoplasia.

The coronal portion of the collected teeth were removed from the radicular portion with a slow speed diamond saw (Laizhou Weiyi Co. Ltd Model DTQ-5) with water irrigation. The labial portion of the section tooth was then divided longitudinally resulting in 60 samples obtained from a total of 30 teeth. The labial enamel surfaces of the specimens were grinded using silicon carbide paper (grades 600-1200) under water irrigation to produce flat surfaces. The specimens were then placed in pre-formed
Teflon molds (10mmx $8 \mathrm{~mm} \times 2 \mathrm{~mm})$ and finally fixated into position with flow able composite resin.

\subsection{Demineralizing Protocol}

A carbonated drink (Coca Cola Pakistan Ltd) was chosen as a demineralizing bath to induce dental erosion. The $\mathrm{PH}$ of the drink at $20^{\circ} \mathrm{C}$ was measured to be at 2.4

All the samples were then randomly assigned to one of the following three groups:

GROUP 1: 20 SAMPLES: CONTROLLED GROUP (untreated)

GROUP 2: 20 SAMPLES: DEMINERALIZATION (4 cycles of 2-minute immersion [26]

GROUP 3: 20 SAMPLES: DEMINERALIZATION (4 cycles of 2-minute immersion followed by 7 days' immersion in CESP)

The subsurface enamel erosion was induced by placing the tooth samples from groups 2 and 3 in $6 \mathrm{ml}$ of soft drink for 2 mins each at room temperature. Four consecutive cycles of immersion were carried out at six hours interval before and after cleaning with deionized water [18]. Samples from group 3 were then placed in CESP for 21 hours for consecutive 7 days to induce remineralization.

\subsection{AFM Observations}

Surface roughness assessment images were taken with Atomic Force Microscopy Auto probe CP100 equipped with piezoelectric scanner of tapping mode. The root mean square Rrms was calculated from at least ten different film areas with a resolution of 256x256 pixels.

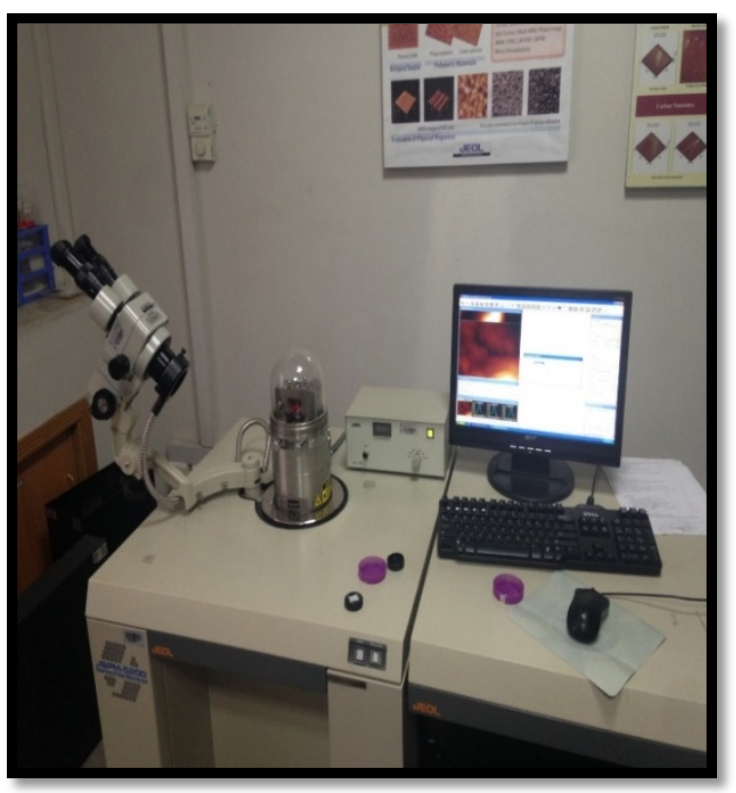

Figure 1. Atomic Force Microscope

\subsection{Statistical Analysis}

Data was analyzed by using SPSS 11.0 software. The differences in the mean roughness values between the three groups were analyzed by using ANOVA. Tuckey's post hoc tests were performed to reveal the differences among the 3 groups. 


\section{Results}

Tests were run by using SPSS on the following data

\begin{tabular}{|c|c|c|}
\hline $\begin{array}{c}\text { CONTROL } \\
\text { GROUP 1 }\end{array}$ & $\begin{array}{c}\text { DEMINERALIZED } \\
\text { GROUP 2 }\end{array}$ & $\begin{array}{c}\text { REMINERALIZED } \\
\text { GROUP 3 }\end{array}$ \\
\hline $\mathbf{0 . 0 4}$ & 0.26 & 0.14 \\
\hline $\mathbf{0 . 0 6}$ & 0.22 & 0.16 \\
\hline $\mathbf{0 . 0 3}$ & 0.38 & 0.13 \\
\hline $\mathbf{0 . 0 8}$ & 0.21 & 0.17 \\
\hline $\mathbf{0 . 0 9}$ & 0.31 & 0.12 \\
\hline $\mathbf{0 . 0 4}$ & 0.29 & 0.14 \\
\hline $\mathbf{0 . 0 6}$ & 0.36 & 0.17 \\
\hline $\mathbf{0 . 0 8}$ & 0.24 & 0.14 \\
\hline $\mathbf{0 . 0 6}$ & 0.38 & 0.13 \\
\hline $\mathbf{0 . 0 5}$ & 0.34 & 0.12 \\
\hline $\mathbf{0 . 0 6}$ & 0.29 & 0.14 \\
\hline $\mathbf{0 . 0 7}$ & 0.28 & 0.15 \\
\hline $\mathbf{0 . 0 8}$ & 0.26 & 0.12 \\
\hline $\mathbf{0 . 0 6}$ & 0.32 & 0.16 \\
\hline $\mathbf{0 . 0 4}$ & 0.30 & 0.18 \\
\hline $\mathbf{0 . 0 6}$ & 0.38 & 0.14 \\
\hline $\mathbf{0 . 0 7}$ & 0.29 & 0.16 \\
\hline $\mathbf{0 . 0 5}$ & 0.38 & 0.15 \\
\hline $\mathbf{0 . 0 6}$ & 0.26 & 0.13 \\
\hline $\mathbf{0 . 0 4}$ & 0.29 & 0.14 \\
\hline & & \\
\hline
\end{tabular}

The mean roughness values have been shown in Table 1 . One Way Anova analysis showed a statistically significant difference with a p-value of 0.00 , between the mean roughness values of the three groups. Tuckey's post hoc test revealed that there is a statistically significant difference $(0.158 \pm 0.012)$ between the mean roughness values of Group 2 and Group 3 as well as between Group 1 and Group3 $(0.085 \pm 0.012)$ with a p-value of 0.00 , indicating that following treatment by CESP the mean roughness values decreased significantly.
Table 1. Mean Roughness Values (Rrms) obtained in three groups

\begin{tabular}{|l|l|c|}
\hline GROUPS & \multicolumn{1}{|c|}{ TREATMENT } & Rrms \\
\hline Group 1 & Control (no treatment) & $0.059 \pm 0.016$ \\
\hline Group 2 & Demineralization Only & $0.302 \pm 0.054$ \\
\hline Group 3 & $\begin{array}{l}\text { Demineralization followed by 7 days' } \\
\text { immersion in CESP }\end{array}$ & $0.145 \pm 0.018$ \\
\hline
\end{tabular}

Figure 2(a) shows untreated enamel surfaces (Group 1). Figure 2(b) shows tooth surfaces placed in a demineralizing bath (Group 2). Figure 2(c) reveals a specimen with surfaces demineralized and then exposed to the protective effect of CESP. The enamel surfaces of the untreated teeth appeared quite smooth Figure 2(a) whereas, apparent increase of surface roughness was observed after exposure to soft cola drink Figure 2(b) After Application of remineralizing solution all tooth specimens showed reduction in Rrms values as shown in Figure 2(c).

\section{Discussion}

The protective effect of CESP using AFM has not been addressed in literature till now. AFM was first used to study the effects of bleaching agent on tooth surfaces by Hegedus et al [19] who compared the images obtained from AFM with those obtained with SEM. Unlike, SEM dehydrated enamel surfaces can be studied with AFM which gives high resolution and contrast images making it an important modern tool to study the remineralizing effects of CESP on eroded enamel surfaces [19].

Tapping mode AFM (TM AFM) images were used to study the topographic features of polished tooth surfaces and to show net differences between exposed and un exposed enamel surfaces. AFM based Nano indentation is useful to identify the demineralized and re mineralized enamel surfaces with high accuracy [6].

The protective effects of chicken egg shell powder solution on enamel was compared with samples treated with demineralizing solution and the results showed a statistically significant difference $(0.158 \pm 0.012)$ with a $p$ value of 0.00 indicating the effectiveness of remineralizing effect of CESP on enamel surfaces.
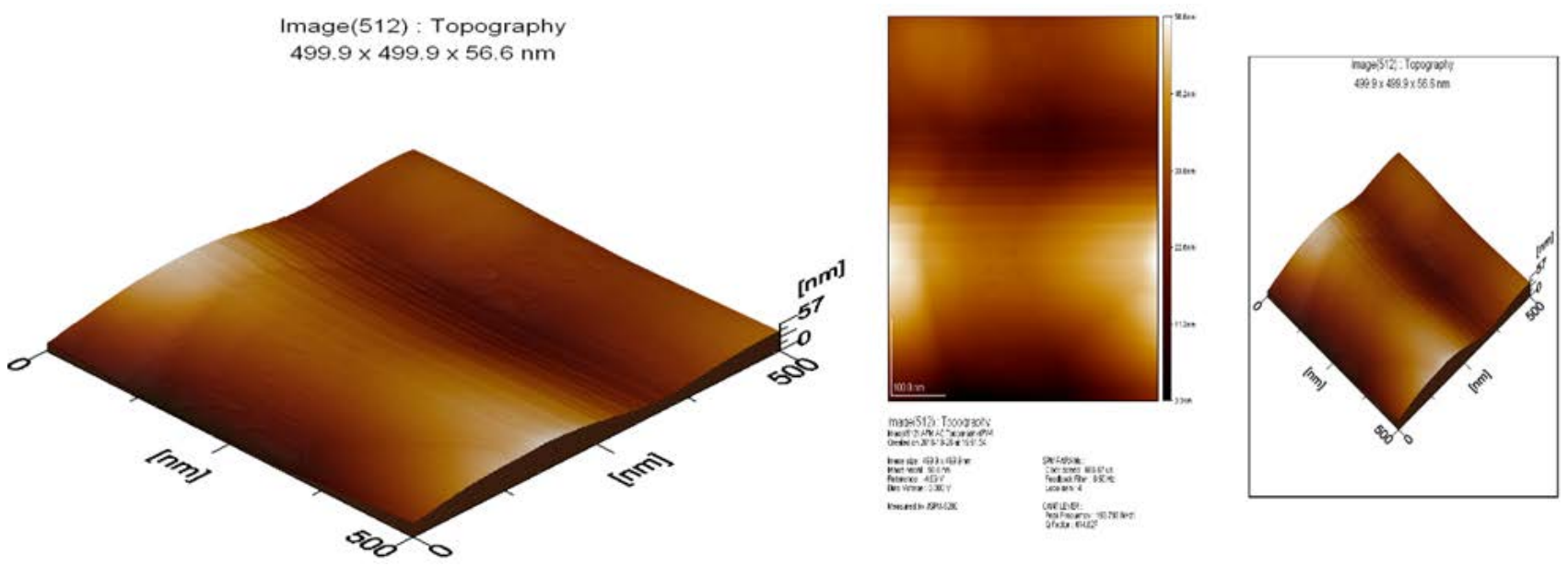

Figure 2. (a) -2-D and 3-D Images of untreated specimens surface (group 1) 

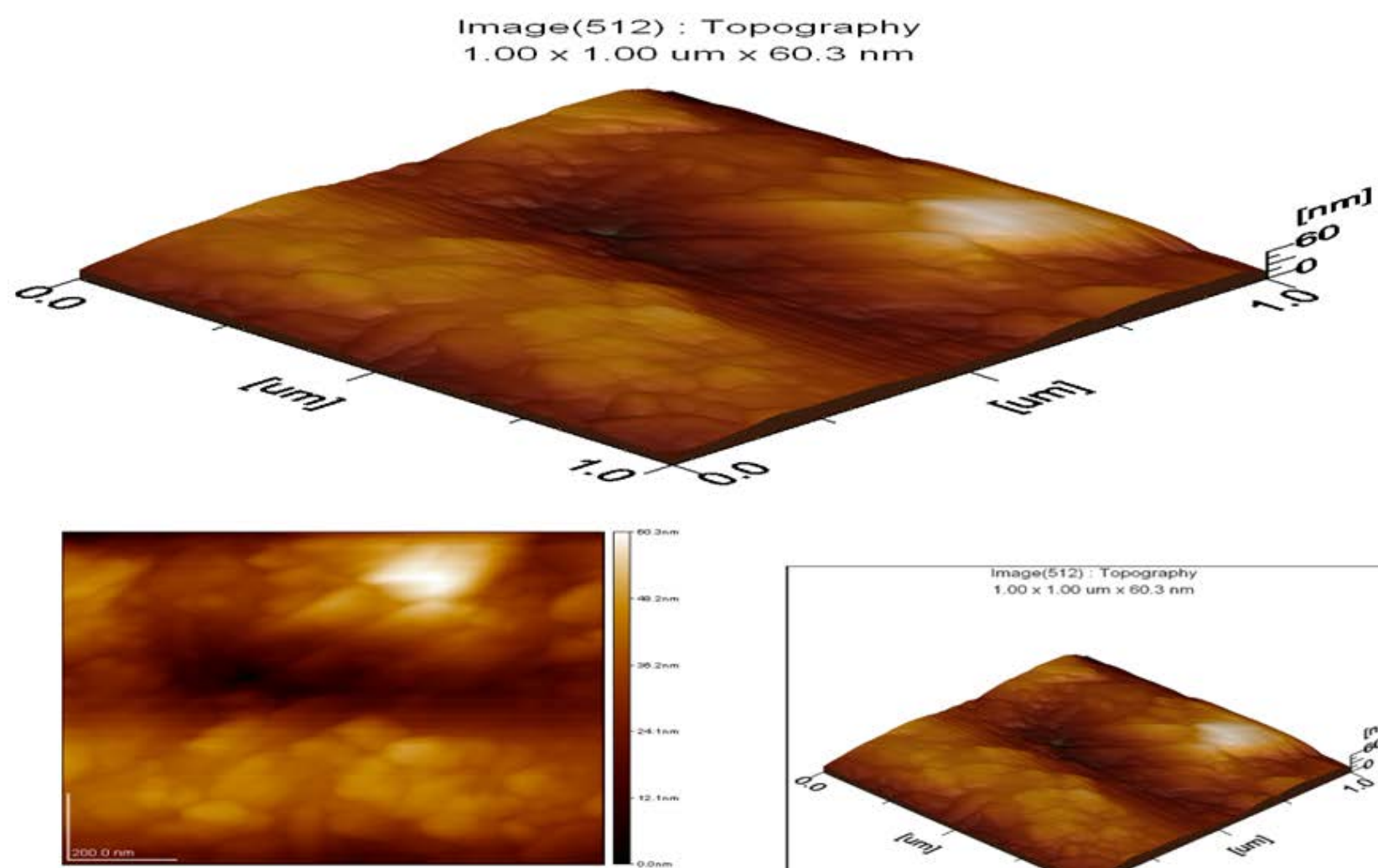

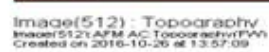

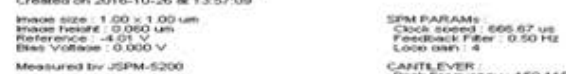

FAтreves

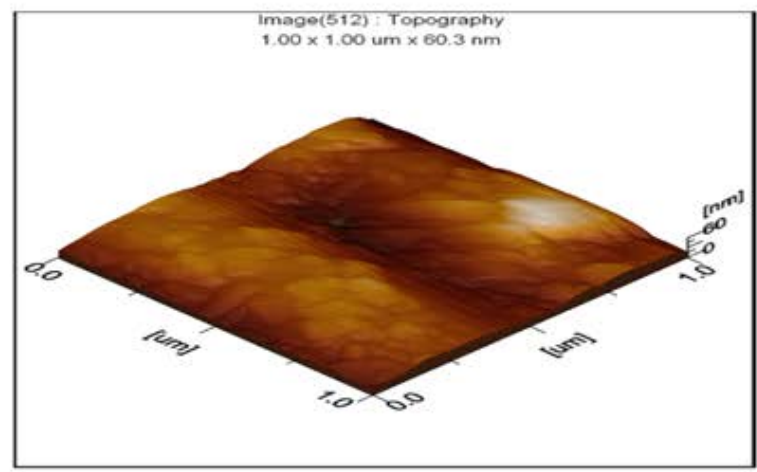

Image(512): Topography

$2.00 \times 2.00 \mathrm{um} \times 85.4 \mathrm{~nm}$
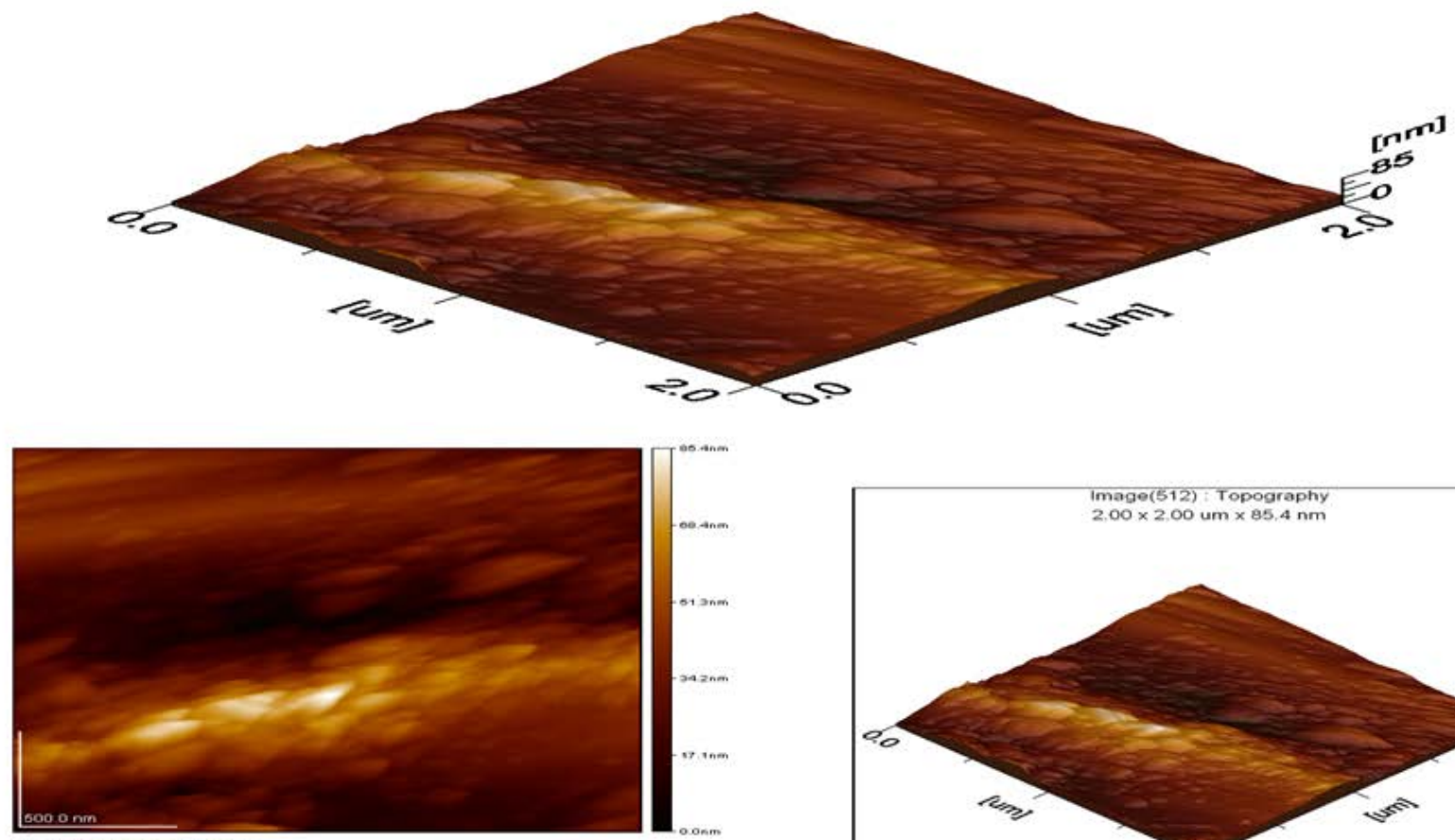

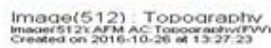

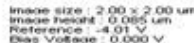
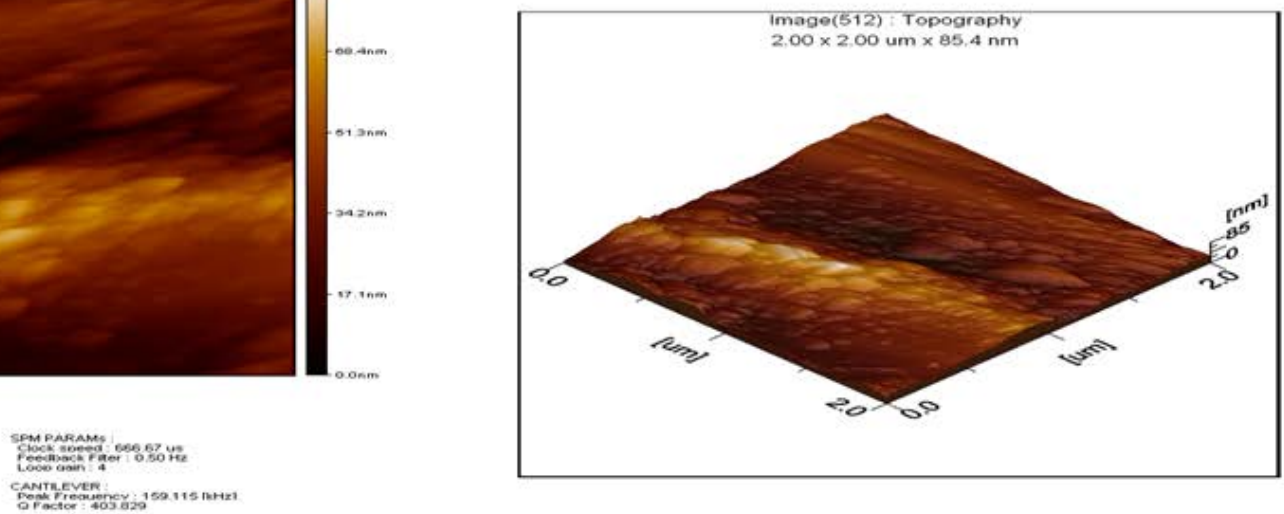

Figure 2. (b)-2-D and 3-D Images of specimen surfaces after demineralization with soft drink (group 2)

Enamel subsurface is less mineralized than enamel surface which is more frequently seen on the deciduous teeth but can also be found on the permanent tooth surfaces. Polished tooth samples used in the study to minimize the natural variation of surface enamel between different teeth which may respond differently to acid dissolution [20]. However, polished tooth surfaces show more erosion as compare to natural tooth surfaces in the oral cavities [21]. 

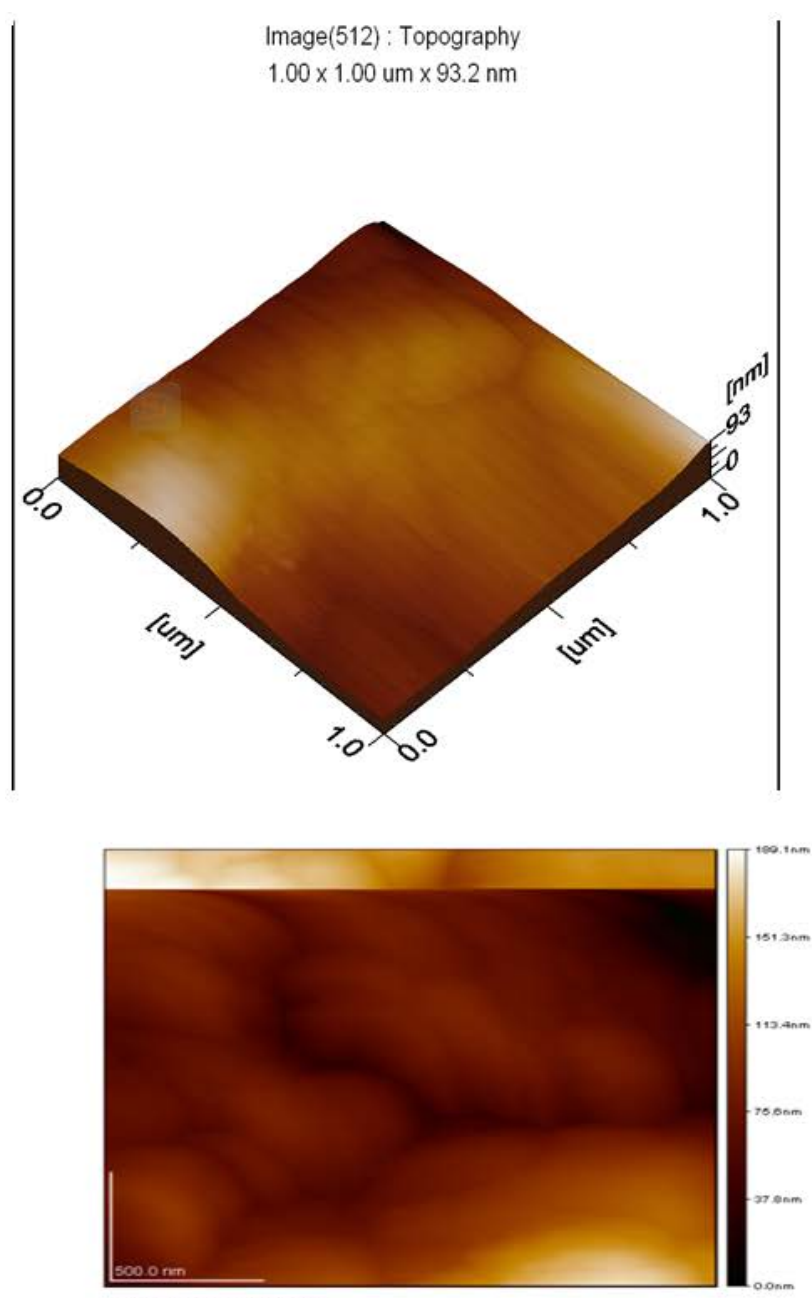

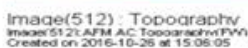

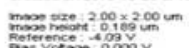

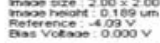

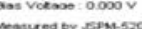

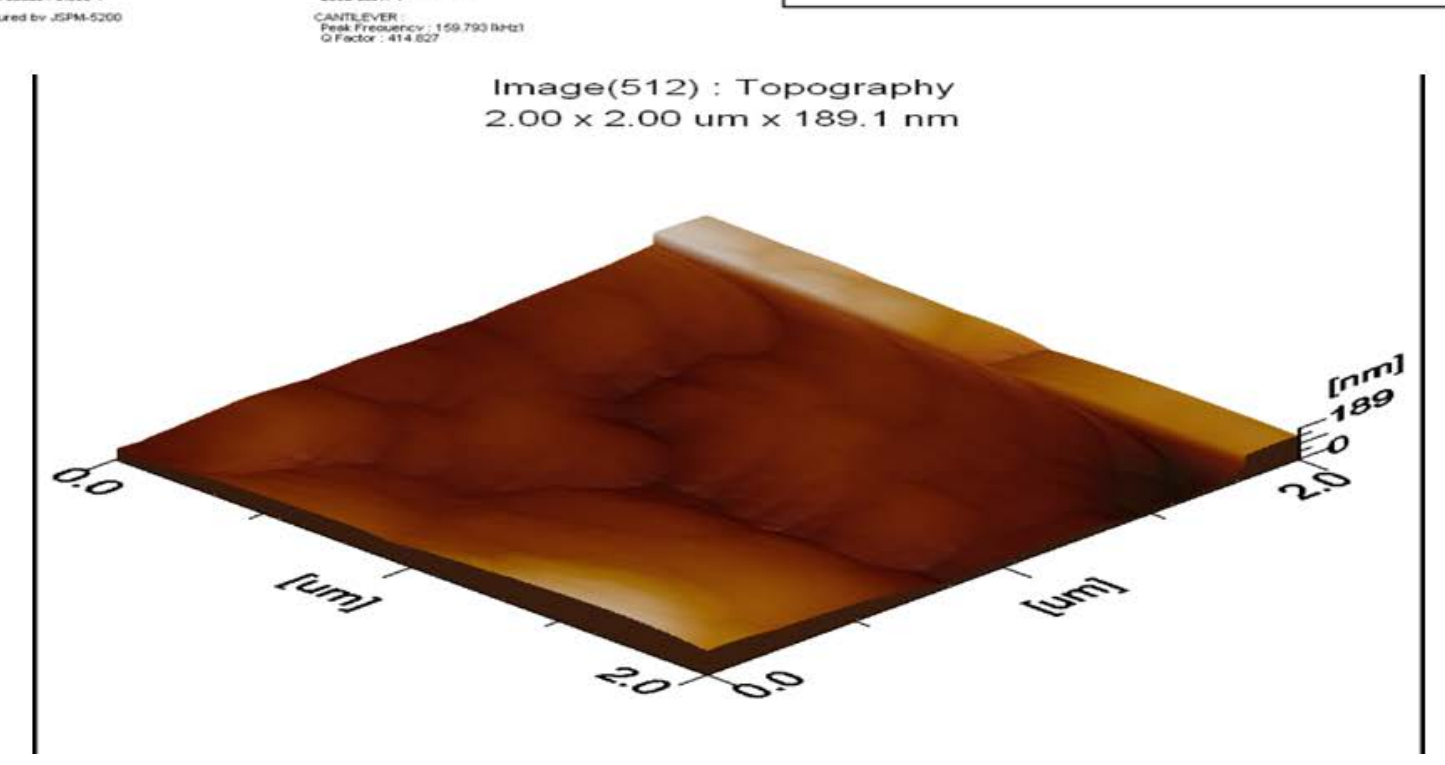

Figure 2. (c)-2-D and 3-D Images of specimen surfaces after remineralization with CESP (group 3)

In this study, one of the most commonly consumed carbonated beverage Coca Cola (Pak Ltd) was chosen to induce enamel surface erosion. A new beverage was used for each specimen and measured at $6 \mathrm{ml}$ for all samples. A constant temperature of $20^{\circ} \mathrm{C}$ was kept as there appears to be relationship between erosion and temperature of beverages [22]. Moreover, it is replenished for every
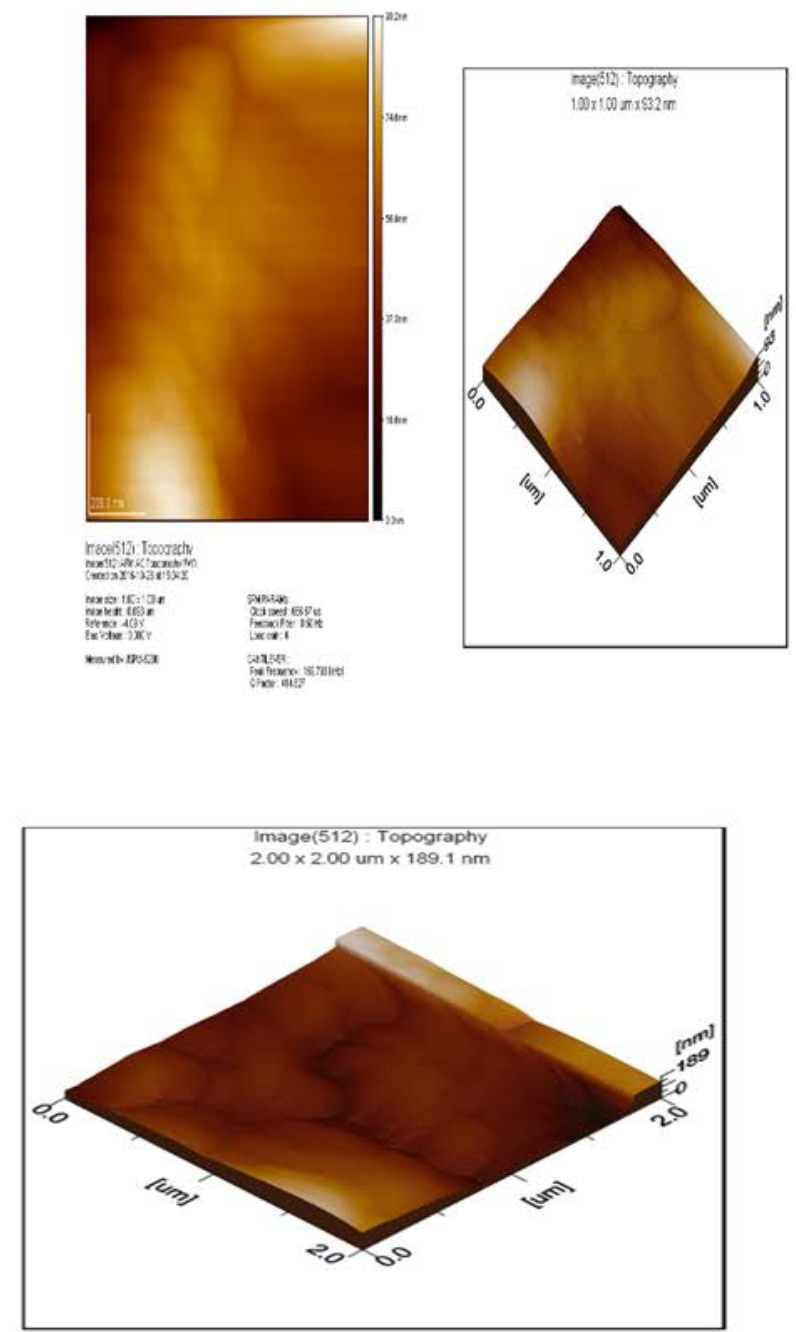

2 mins to reduce the buffering effect produced by the ionic dissolution from the surface enamel [18].

Eggs shell forming the outer crust of an egg is a non-edible product, mostly disposed of as a waste. In recent years, its use in various fields has been widely investigated $[11,12,13]$. Due to its high calcium content it has the potential to use as human dietary calcium supplement 
especially for post-menopausal women and elderly population [11].

X- Ray fluorescence spectroscopic analysis of CESP revealed that it contains about $98 \%$ Calcium, $0.46 \%$ of phosphate, $0.53 \%$ of Magnesium, $0.18 \%$ of Strontium, $0.03 \%$ of Potassium [23].This high concentration of bio available Ca plays a vital role in enamel remineralization when CESP applied topically [23]. Chicken egg shell powder had gone through the process of calcination to remove pathogens and to increase its alkalinity. Addition of acetic acid (10\%) ensured that powder is virtually free of pathogens [17]. $\mathrm{PH}$ of CESP solution measured by $\mathrm{pH}$ meter is 11.2. This greater $\mathrm{pH}$ is favorable for the increased ionic activity of anions such as hydroxyl ions and phosphate ions. Thus more ions are available for enamel surface remineralization. If the $\mathrm{pH}$ of remineralizing solution is low (acidic)there will be more concentration of $\mathrm{H}+$ ions which combines with the available anions and thus less ions will be available for remineralization.

Availability of calcium and phosphate ions is essential for remineralization to occur and increased $\mathrm{pH}$ of the solution along with rich bioavailability of phosphate and calcium ions is mainly involved for the process of remineralization [24]. Thus by maintaining the state of supersaturation of these Calcium and phosphate ions promotes remineralization and depresses the process of erosion on tooth surfaces [22]. CESP application results in the remineralization due to rich bioavailability of calcium as well as phosphate ions along with increased $\mathrm{pH}$.

Due to the natural source and easy bioavailability CESP has a promising future in treating eroded enamel surfaces. However, to further increase the remineralizating ability more extensive clinical studies are required to improve its remineralization potential comparable to the various commercially available agents.

\section{Conclusion}

Within the limitations of this in vitro study it can be concluded that

1. All the samples showed an increase in surface roughness value after exposure to carbonated drink.

2. Reduction in surface roughness has been observed in all tooth samples after application of CESP.

3. Protective effects of CESP on enamel was compared with samples treated with demineralizing solution and results showed a statistically significant difference indicating the effectiveness of remineralizing effect of CESP on enamel surfaces.

\section{Acknowledgements}

I submit my heartiest gratitude to Dr Faisal Moeen for the most dedicated guidance, encouragement, patience and support for this research. Dr Saleha Nisar whose assistance and corporation was essential for the competion of this project. National University of Science and Technology, Islamabad, Pakistan for the use of Atomic Force Microscope.

\section{Statement of Competing Interests}

The authors have no competing interests.

\section{References}

[1] Murdoch_Kinch CA MM. Minimally invasive dentistry. $J$ Am Dent Assoc.134:87_95. 2003.

[2] R. PJO. Effects of potassium ions on action potential conduction in A- and C- fibers of rat spinal nerves J Dent Res 74(2): 634-641. 1995.

[3] Peterson GBD. The caries decline: a review of reviews. Eur J Oral Sci 1996; 104: 436-43.

[4] A. L. Erosive tooth wear_ a multifactorial condition of growing concern and increasing knowledge. Monogr Oral Sci 20:1-8.2006.

[5] Jaeggi TLA. Prevalence, Incidence and distribution of erosion. Monogr Oral Sci 20: 44-65. 2006.

[6] Poggio C LM, Dagna A, Chiesa M, Bianchi S. "Protective effect on enamel demineralization of a CPP-ACP paste: An AFM invitro study ". J Dent 37: 949-954. 2009.

[7] Padma Gandi MS, Sangeeta Meena and Pallavi Waghmare. "Comparative Study on remineralizing ability of Casein phosphopeptide Amorphous calcium phosphate and b-tricalcium phosphate on dental erosion: An invitro AFM Study". Gandi et al, Dentistry, 5: 8. 2015.

[8] Oshiro M YK, Takamizawa T,Inage H,et al. "Effect of CPP-ACP paste on tooth mineralization: an FE-SEM study". J Oral Sci49: 115-20. 2007

[9] Kato MT LM, Sales-Pares SH, Buzalaf MA. "Preventive effect of commercial desensitizing toothpastes on bovine enamel erosion in vitro". Caries Res, 44: 85-9, 2010.

[10] Reynolds EC. "Remineralization of enamel subsurface lesions by casein phosphopeptide -stabilization calcium phosphate solutions", J Dent Res, 79(9): 1587-95; 1997.

[11] Schaafsma A vDJ, Muskiet FA, Hofstede GJ, Pakan I, van der Veer E. "Positive effects of a chicken eggshell powder -enriched vitamin-mineral supplement on femoral neck bone mineral densityin healthy late post-menopausal Dutch women". Br J Nutr; 87(3): 267-75. 2002.

[12] Park JW BS, Suh JY, Lee DH, Kim SH, Kim H, et al. "Evaluation of bone healing with eggshell-derived bone graft substitutes in rat calvaria: a pliot study". J Biomed Mater Res A, 87(1). 203-14. 2008.

[13] Rovensky J SM, Masaryk P, Svik K, Istok R. "Eggshell calcium in the prevention and treatment of osteoprosis". Int J Clin Pharmacol Res, 23(2-3). 83-92. 2003.

[14] Farina M SA, Weissmuller G, Cruz R, Kachar B, Bisch PM. "Atomic force microscopy study of tooth surfaces". Journal of Structural Biology, 125: 39-49. 1999.

[15] Finke M JK, Parker DM. "The early stages of native enamel dissolution studied with atomicc force microscopy". Journal of colloid and interface Science; 232: 156-64. 2000.

[16] Methods of producing egg shell powder: Patents: US 20060062857 A 1.

[17] Shen P MD, Cochrane NJ,Walker GD,Yuan Y,Reynolds C,et al. "Effect of added calcium phosphate on enamel remineralization by fluoride in a randomized controlled in situ trial". J Dent; 39: 518-25. 2011.

[18] Tantbirojin D HA, Ericson MD, Poolthong S. "Change in surface hardness of enamel by a cola drink and a CPP-ACP paste". Journal of Dentistry; 36: 74-9. 2008.

[19] Hegedus C BT, Flora-Nagy F, Keszthelyi G, Jenei A. "An atomic force microscopy study on the effect of bleaching agents on enamel surface". J Dent ; 27: 509-15.1999.

[20] Adebayo OA BM, Tyas MJ. "An SEM evaluation of conditioned and bonded enamel following carbamide peroxide bleaching and casein phosphopeptide-amorphous calcium phosphate (CPP-ACP) treatment". J Dent; 37: 297-306. 2009.

[21] Ranjitkar S RJ, Kaidonis JA, Richards LC, Townsend GC, Bartlett DW. The effect of casein phospho-peptide-amorphous calcium phosphate on erosive enamel and dentin wear by toothbrush abrasion. J Dent; 37: 250-54. 2009. 
[22] Barbour ME RJ. The laboratory assessment of enamel erosion: a review. Journal of Dentistry.; 32: 591-602. 2004.

[23] Bejoy Mony AVRE, Mohamed Fayas Ghani, Ashwin Narayanan, Anand S., Ajit george mohan. Effect of Chicken Egg Shell Powder Solution on Early Enamel Carious Lesions: An Invitro Preliminary Study.

[24] Hicks J G-GF, Flaitz C. Biological factors in dental caries enamel structure and the caries process in the dynamic process of demineralization and remineralization (part 2). J Clin Pediatr Dent ; 28: 119-24. 2004.

[25] C. G-C. The chemistry of caries: remineralization and demineralization events with direct clinical relevance. Dent Clin North Am; 54(3): 469-78. 2010.

[26] Poggio, Claudio, et al. "Impact of two toothpastes on repairing enamel erosion produced by a soft drink: an AFM in vitro study". Journal of dentistry 38(11): 868-874. 2010. 